\title{
Letter to the Editor concerning "Osteolysis after cervical disc arthroplasty" by Joaquim AF, et al. (Eur Spine J; [2020]: doi: 10.1007/ s00586-020-06,578-2)
}

\author{
Ting-kui Wu ${ }^{1} \cdot$ Qing-yu Liu ${ }^{2}$ Junbo He ${ }^{1} \cdot$ Bei-yu Wang ${ }^{1} \cdot$ Hao Liu ${ }^{1}(\mathbb{C}$
}

Received: 4 September 2020 / Revised: 4 September 2020 / Accepted: 15 September 2020 / Published online: 25 September 2020

(c) Springer-Verlag GmbH Germany, part of Springer Nature 2020

With great interest, we read the article titled "Osteolysis after cervical disc arthroplasty" written by Joaquim et al. in European Spine Journal [1]. The authors conducted a systematic literature review of osteolysis after cervical disk arthroplasty (CDA) and discussed the causes, incidence, and management. We highly appreciate their contribution to this topic; however, some issues in the article may mislead the readers, which needs further clarification.

First, bone resorption may be a different phenomenon of the osteolysis after CDA. Bone resorption tends to be a natural physiological change that favors the early bone remodeling process [2] and follows a stable radiographic feature. In contrast, osteolysis is a long-term pathological process of progressive destruction of periprosthetic bone, characterized as a progressive radiolucent line or cavitation at the implant-bone interface on X-rays and CT scans [3]. The authors mentioned anterior bone loss following CDA in their article was mainly pertaining to bone remodeling rather than osteolysis. The two concepts should not be confused.

Second, we noticed that two of the screened articles were from the same medical center and the same follow-up point [1]. However, the authors included and analyzed them separately, which may cause inaccurate conclusions. We suggest that the authors should re-screen the original articles or analyze the two articles together.

Third, the proposed classification of bone loss was less clinically instructive. Although there was still no consensus on the classification system of bone loss, most of the current classification systems were based on the radiographic features. According to the published articles and our findings

Hao Liu

dr.liuhao6304@hotmail.com

1 Department of Orthopedic Surgery, West China Hospital, Sichuan University, No. 37 Guo Xue Rd, Chengdu 610041, Sichuan, China

2 West China School of Medicine, Sichuan University, No. 37 Guo Xue Rd, Chengdu 610041, China
[4], none of them reported a relative motion between prosthesis and endplates during extension-flexion motion even with severe bone loss. Therefore, it is not appropriated for motion between prosthesis and endplates as an evaluation index. Furthermore, very few patients after CDA suffered more than $20 \%$ anterior bone loss at the arthroplasty segment, and some patients with only more than $10 \%$ anterior bone loss may occur endplate collapse. Thus, their proposed bone loss classification system was difficult to distinguish patients who really needed a further monitor.

Once again, we congratulate the authors for their hard work and hope that the readers may benefit from it.

\section{Compliance with ethical standards}

Conflict of interest The authors declare that they have no conflict of interest.

\section{References}

1. Joaquim AF, Lee NJ, Lehman RA Jr, Tumialán LM, Riew KD (2020) Osteolysis after cervical disc arthroplasty. Eur Spine J. https://doi.org/10.1007/s00586-020-06578-2

2. Li Z, Kuhn G, von Salis-Soglio M, Cooke SJ, Schirmer M, Muller R, Ruffoni D (2015) In vivo monitoring of bone architecture and remodeling after implant insertion: the different responses of cortical and trabecular bone. Bone 81:468-477. https://doi. org/10.1016/j.bone.2015.08.017

3. Kurcz B, Lyons J, Sayeed Z, Anoushiravani AA, Iorio R (2018) Osteolysis as it pertains to total hip arthroplasty. Orthoped Clin North Am 49(4):419-435. https://doi.org/10.1016/j.ocl.2018.06.001

4. Wu TK, Liu H, Wang BY, He JB, Ding C, Rong X, Yang Y, Huang KK, Hong Y (2020) Incidence of bone loss after Prestige-LP cervical disc arthroplasty: a single-center retrospective study of 396 cases. Spine J Off J North Am Spine Soc 20(8):1219-1228. https ://doi.org/10.1016/j.spinee.2020.05.102

Publisher's Note Springer Nature remains neutral with regard to jurisdictional claims in published maps and institutional affiliations. 